\title{
Premotor Cortical-Cerebellar Reorganization in a Macaque Model of Primary Motor Cortical Lesion and Recovery
}

\author{
(1)Tatsuya Yamamoto, ${ }^{1,2}$ Takuya Hayashi, ${ }^{3}$ Y Yumi Murata, ${ }^{2}$ Takayuki Ose, ${ }^{3}$ and ${ }^{-}$Noriyuki Higo $^{2}$ \\ ${ }^{1}$ Faculty of Medical and Health Sciences, Tsukuba International University, Tsuchiura, Ibaraki, 3000051, Japan, ${ }^{2}$ Human Informatics Research Institute, \\ National Institute of Advanced Industrial Science and Technology, Tsukuba, Ibaraki, 3058568, Japan, and ${ }^{3}$ Laboratory for Brain Connectomics Imaging, \\ RIKEN Center for Biosystems Dynamics Research, Kobe, Hyogo, 6500047, Japan
}

\begin{abstract}
Neuromotor systems have the capacity for functional recovery following local damage. The literature suggests a possible role for the premotor cortex and cerebellum in motor recovery. However, the specific changes to interactions between these areas following damage remain unclear. Here, we demonstrate potential rewiring of connections from the ipsilesional ventral premotor cortex (ip-PMv) to cerebellar structures in a nonhuman primate model of primary motor cortex (M1) lesion and motor recovery. Cerebellar connections arising from the ip-PMv were investigated by comparing biotinylated dextran amine (BDA) between two groups of male Macaca mulatta: M1-lesion/motor recovery group and intact group. There were more BDA-labeled boutons and axons in all ipsilesional deep cerebellar nuclei (fastigial, interposed, and dentate) in the M1-lesion/recovery group than in the intact group. The difference was evident in the ipsilesional fastigial nucleus (ip-FN), and particularly observed in its middle, a putative somatosensory region of the ip-FN, which was characterized by absent or little expression of aldolase C. Some of the altered projections from the ip-PMv to ip-FN neurons were confirmed as functional because the synaptic markers, synaptophysin and vesicular glutamate transporter 1, were colocalized with BDA-labeled boutons. These results suggest that the adult primate brain after motor lesions can reorganize large-scale networks to enable motor recovery by enhancing sensorimotor coupling and motor commands via rewired fronto-cerebellar connections.
\end{abstract}

Key words: biotin dextran amine; cerebellar compartmentalization; fastigial nucleus; monkey; neural circuits; plasticity

Significance Statement

Damaging the motor cortex causes motor deficits, which can be recovered over time. Such motor recovery may result from functional compensation in remaining neuromotor areas, including the ventral premotor cortex. We investigated compensatory changes in neural axonal outputs from ventral premotor to deep cerebellar nuclei in a monkey model of primary motor cortical lesion and motor recovery. The results showed an increase in premotor projections and synaptic formations in deep cerebellar nuclei, especially the sensorimotor region of the fastigial nucleus. Our results provide the first evidence that large-scale reorganization of fronto-cerebellar circuits may underlie functional recovery after motor cortical lesions.

\section{Introduction}

Neuromotor systems have the capacity for functional recovery following local damage, but the mechanisms underlying re-

Received Jan. 8, 2019; revised Sept. 2, 2019; accepted Sept. 6, 2019.

Author contributions: T.Y., T.H., Y.M., and N.H. designed research; T.Y., T.H., Y.M., T.O., and N.H. performed research; T.H. contributed unpublished reagents/analytic tools; T.Y., T.H., Y.M., T.O., and N.H. analyzed data; T.Y., T.H., and N.H. wrote the paper.

This work was supported by a Grant-in-Aid for Scientific Research (B) (16H03300 to T.H.) and Young Scientists (B) (18K17683 to T.Y) from the Ministry of Education, Culture, Sports, Science, and Technology in Japan. We thank M. Yamaguchi, A. Yoshida, and Y. Yoshida for excellent technical assistance; Dr. H. Onoe, Dr. K. Sawada, and Dr. R. Hawkes for excellent technical advice; and Editage (www.editage.jp) for English language editing.

The authors declare no competing financial interests.

Correspondence should be addressed to Takuya Hayashi at takuya.hayashi@riken.jp or Noriyuki Higo at n.higo@aist.go.jp.

https://doi.org/10.1523/JNEUROSCI.0077-19.2019

Copyright $\odot 2019$ the authors covery are not fully understood. Experimental animal studies provide information essential for understanding brain mechanisms underlying functional recovery from brain damage.

Lesioning of the primary motor cortex (M1) in nonhuman primates has been an established experimental model of motor recovery for over a century (Leyton and Sherrington, 1917; Glees and Cole, 1950; Travis, 1955; Passingham et al., 1983; Nudo et al., 1996; Friel and Nudo, 1998; Liu and Rouiller, 1999; for review, see Vilensky and Gilman, 2002; Dancause et al., 2005). Several studies have demonstrated that parts of an undamaged brain may be reorganized in functional recovery after focal damage to M1 (Nudo et al., 1996; Frost et al., 2003; Dancause et al., 2005; for review, see Nudo, 2013). However, it remains poorly understood how local and long-range connectivity changes contribute to motor recovery. 
Table 1. Intact and M1-lesioned monkeys used in the present study

\begin{tabular}{|c|c|c|c|c|c|c|}
\hline \multirow[b]{2}{*}{ Monkeys } & \multicolumn{3}{|l|}{ Intact } & \multicolumn{3}{|l|}{ Lesion } \\
\hline & A & B & C & D & $\mathrm{E}$ & $\mathrm{F}$ \\
\hline Weight, kg & 5 & 5.5 & 4 & 7.5 & 8 & 7 \\
\hline Age, $d$ & 1973 & 1979 & 1895 & 2444 & 2284 & 7341 \\
\hline \multirow{2}{*}{ BDA injection site, mm; AP, ML } & $24.00,23.00$ & $23.00,18.50$ & $22.50,23.00$ & $23.00,20.50$ & $23.00,22.00$ & $21.60,21.60$ \\
\hline & $25.40,24.85$ & $24.40,20.35$ & $23.90,24.85$ & $24.40,22.35$ & $24.40,23.85$ & $23.00,23.45$ \\
\hline Volume of BDA injected, $\mu \mathrm{l}$ & 0.5 & 0.5 & 1 & 0.5 & 0.5 & 0.5 \\
\hline Estimated volume of BDA injection core, $\mathrm{mm}^{3}$ & 147.8 & 52.8 & 121.6 & 75.5 & - & 49.0 \\
\hline No. of BDA axons in cerebral peduncle & 8 & 11 & 15 & 9 & 11 & 2 \\
\hline Estimated volume of $\mathrm{M} 1$ lesional area, $\mathrm{mm}^{3}$ & - & - & - & 54.1 & 57.1 & 55.0 \\
\hline
\end{tabular}

AP, Anterior-posterior; ML, medial-lateral.

We have previously shown that rhesus monkeys administered a lesion in the M1 digit area and trained for a particular set of motor tasks exhibited gradual recovery of dexterous hand movements (Murata et al., 2008), and task-related cerebral blood flow increases in ipsilesional ventral premotor cortex (ip-PMv) during recovery (Murata et al., 2015b). In the same animal model, we also observed increased gene expression of a plasticity-related molecule, growth-associated protein-43, in the excitatory neurons of the ip-PMv, suggesting that efferent connections from the ip-PMv were remodeled for functional recovery (Murata et al., 2015a).

However, there is scarce evidence regarding which ip-PMv neural circuits are reorganized in association with functional motor recovery and how this occurs. Dancause et al. (2005) reported a projection from ip-PMv to somatosensory cortex (area 1/2) in motor-recovered monkeys after lesioning of M1 hand area, but not in control animals. They suggested that novel connections between premotor and sensory cortices were established during motor recovery. Reorganization of sensorimotor circuits during motor recovery is supported by a putative role of the ventral premotor cortex in motor control such as motor action, visualmotor transformation, and selective control of arm movements (e.g., shaping hand posture; Rizzolatti et al., 1988; for review, see Rizzolatti and Luppino, 2001).

It is also intriguing that our previous analysis of phsychophysiological interactions (PPI) revealed increased functional connectivity between the contralesional cerebellar cortex and partially lesioned motor area over the entire period of recovery (Murata et al., 2015b). This suggests that the cerebellum is part of a neural circuit that plays a pivotal role in recovering motor behavior. Considering its key role in motor learning (for review, see Ito, 2008; Longley and Yeo, 2014; De Zeeuw and Ten Brinke, 2015), it is reasonable that the cerebellum is involved in motor recovery in conjunction with the ventral premotor area. In fact, neuroimaging studies in stroke patients have reported involvement of the premotor cortex (Seitz et al., 1998) and cerebellum (JohansenBerg et al., 2002; Small et al., 2002) in recovery of hand movements.

Here, we aimed to investigate whether anatomical projections from ip-PMv to the cerebellum are reorganized in an adult macaque monkey model of M1-lesion and motor recovery. Connections between ip-PMv and cerebellar nuclei were investigated with an anterograde tracer [biotin dextran amine (BDA)] in lesioned monkeys after motor recovery and control intact animals. We observed a specific distribution of axon terminals of ip-PMv, especially in the ipsilesional fastigial nucleus (ip-FN), of motor injury/recovery animals. The functionality of ip-PMv to ip-FN projections was confirmed with multiple-fluorescent staining of synaptic markers: vesicular glutamate transporter 1 (VGLUT1) and synaptophysin. Subsequently, ip-PMv projections were shown to be localized to the Aldolase C (Aldoc)-negative zone of the ip-FN, which is thought to process somatosensory information (Sugihara and Shinoda, 2007; Chung et al., 2009; for review, see Sugihara, 2011), with cerebellar compartmentalization markers: Aldoc (aka, zebrin II) and phospholipase C $\beta 3$ (PLCB3).

\section{Materials and Methods}

Subjects. Three intact rhesus macaque monkeys (Macaca mulatta, male; Monkeys A-C) and three M1-lesioned rhesus macaque monkeys (male; Monkeys D-F) were used to compare connectivity of ip-PMv to cerebellum (Table 1). In addition, two intact rhesus macaque monkeys (male; Monkey G, age: 2284 d, weight: 8.4 kg; Monkey H, 2450 d, $8.4 \mathrm{~kg}$ ) were used to investigate the expression pattern of cerebellar compartmentalization markers in the FN.

Animal housing and ethics. All monkeys were $>5$ years of age and purchased from a local provider. The animal use protocol was approved by the Institutional Animal Care and Use Committee of RIKEN Kobe branch (MA2009-12-7). The monkeys were housed in adjoining individual primate cages allowing social interactions under controlled conditions of humidity, temperature, and light. The animals were monitored daily for their health status by the researchers and animal care staff, and they were given appropriate medication by veterinarians when needed. The housing area was maintained on a $12 \mathrm{~h}$ light/dark cycle, and all the experiments were conducted during the light cycle. Environmental enrichment consisted of multiple commercial toys. A commercial primate diet and fresh fruits were provided daily, and water was provided by a spout in the animal cage. Adequate measures were taken to minimize pain or discomfort in accordance with the Guide for the Care and Use of Laboratory Animals by the National Research Council.

The experimental protocol for motor injury and motor recovery in macaque monkeys followed that used in our previous studies (Murata et al., 2015b). In brief, the monkeys underwent pre-lesion training for a motor task using the procedure described in the Motor behavior task section, followed by topographic motor mapping of the M1 with intracortical microstimulation (ICMS; Fig. 1A). Ibotenic acid was then injected to make neuronal lesions in the digit region of M1. Flaccid paralysis in the contralateral hand occurred after M1 lesion, and dexterous hand movements including precision grip were considerably restored during a month of post-lesion training (Fig. 1B).

Three months after the lesion, when the mean success rate reached a plateau level $>90 \%$, BDA was injected into the ip-PMv region (Monkeys $\mathrm{D}-\mathrm{F}$ ). In the intact monkeys (Monkeys $\mathrm{A}-\mathrm{C}$ ), BDA injection was performed after the success rate reached $100 \%$ in the same task the lesioned monkeys performed in their pre-lesion training. After an incubation period of $>1$ month following BDA injection, brain tissues were removed and analyzed histologically to visualize the synaptic boutons and axons in the distal regions thought to have originated from ip-PMv neurons. The following sections provide the details of the training of the 
A
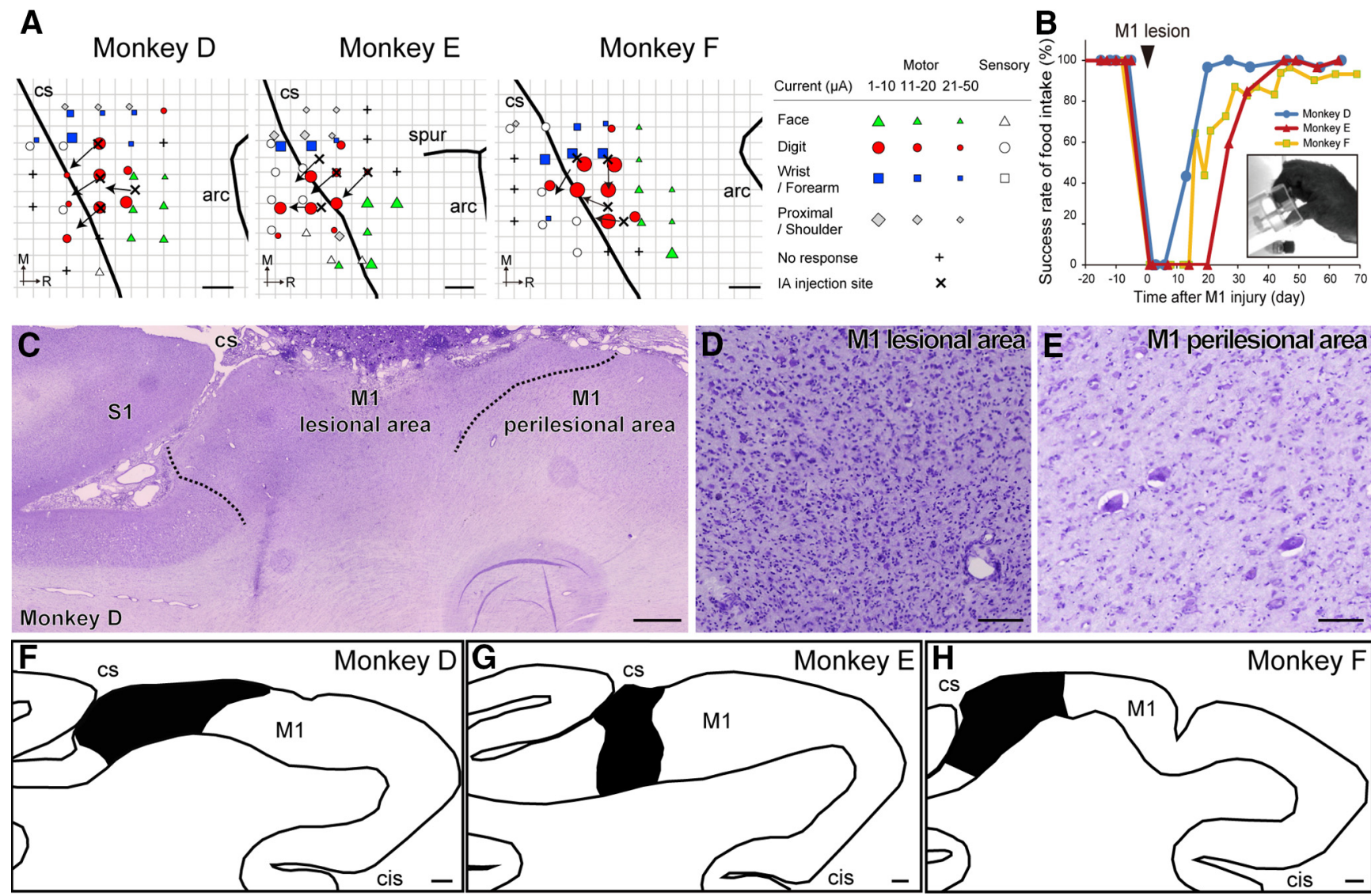

Monkey F

Figure 1. Lesioning of primary M1 hand area and subsequent motor recovery. A, Injection site of ibotenic acid superimposed on ICMS maps of M1 in each lesioned monkey. Arrows indicate the orientation of the blade face at the needle tip. Movement elicited at the threshold and the sensory response to light tactile stimuli is indicated by the symbols. Some electrode penetration sites showed no response to either ICMS at current strengths $\leq 50 \mu \mathrm{A}$ or sensory stimulation. $\boldsymbol{B}$, Time course of changes in the success rate of precision grip in the lesioned monkeys. A successful trial was defined as the removal of the object by using the precision grip between the tip of the index finger and thumb, as shown in the picture. $\boldsymbol{C}-\boldsymbol{E}$, Nissl-stained section of Monkey $\mathbf{D}$ showing a representative lesion of $\mathrm{M} 1$. Ibotenic acid injection in M1 resulted in gliosis and loss of neurons (D), whereas neurons were observed in the neighboring area, defined as the perilesional area. Dotted lines indicate the boundaries between the lesional area and the perilesional area. $\boldsymbol{F}-\boldsymbol{H}$, Line drawing of the $\mathrm{M} 1$ hand area in each lesioned monkey. Black region indicates the lesional area. $C \mathbf{S}$, (entral sulcus; arc, arcuate sulcus; spur, spur of arcuate sulcus; $M$, medial; $R$, rostral; IA, ibotenic acid; $S 1$, primary sensory area; cis-, cingulate sulcus. $S$ Sale bars: $\boldsymbol{A}, 2 \mathrm{~mm} ; \boldsymbol{C}, \boldsymbol{F}-\boldsymbol{H}, 1 \mathrm{~mm} ; \boldsymbol{D}, \boldsymbol{E}, 300 \mu \mathrm{m}$.

motor behavioral task, surgery for ICMS and lesioning, tracer (BDA) injections, histological preparation, and data analysis.

Motor behavior task. For the motor behavior task, we used a small knob $(7 \times 7 \times 7 \mathrm{~mm})$ on a 20 -mm-diameter disk, to the other side of which a piece of sweet potato was attached. Animals could thereby retrieve the potato through a narrow vertical slit (10 mm width; Fig. $1 B$, inset), as detailed in our previous paper (Murata et al., 2015b). Performance of hand movements was also evaluated daily by means of a small object retrieval task using the same procedure as that used in our previous study. The test session to evaluate hand performance consisted of 20 trials.

In addition, the monkeys underwent daily post-lesion training after the $\mathrm{M} 1$ lesion for $>30 \mathrm{~min}$ a day, $4 \mathrm{~d}$ a week, with the retrieval task. Moreover, during the post-lesion training period (from Day 1 to Days 57, 56 , and 62 after the M1 lesion for Monkeys D-F, respectively), the monkeys wore a jacket with a dead-end sleeve covering the unaffected hand (Alice King Chatham Medical Arts; Lomir Biomedical), which allowed them to use the affected hand, as in our previous studies (Murata et al., 2008; Sugiyama et al., 2013). This method resembles that used in constraint-induced movement therapy, widely adopted in the rehabilitation of stroke patients (Taub et al., 2002). Grip movement was recorded with a CCD camera (XC-HR50, Sony) and Windows Live Movie Maker 2011 (Microsoft) was used for video analysis.

Lesion induction. Experimental lesioning of the hand digit area of M1 was induced with the same procedure as reported in our previous papers (Murata et al., 2008, 2015b). Topographic motor maps of M1 were constructed with the ICMS technique (Fig. 1A). Under sterile conditions and pentobarbital anesthesia $(25 \mathrm{mg} / \mathrm{kg})$, a craniotomy was conducted over
M1. Subsequently, a stainless-steel chamber and head holders were affixed to the skull with dental acrylic. ICMS was conducted under ketamine anesthesia, with the rate of ketamine infusion adjusted to maintain relatively stable anesthesia. A flexible tungsten microelectrode (MicroProbe) was advanced perpendicular to the cortical surface to a depth of $5-15 \mathrm{~mm}$ at intervals of $500 \mu \mathrm{m}$ with a hydraulic microdrive. A conventional ICMS technique (1-100 $\mu \mathrm{A}, 200 \mu \mathrm{s}$ in duration, 12 pulses, monophasic cathodal pulses at $333 \mathrm{~Hz}$ ) was used to evoke movement at each electrode penetration site.

Electrophysiological recording was also conducted to determine the border between the primary motor and sensory areas by using a head amplifier and main amplifier. The sensory response was investigated by applying light tactile stimuli to the face, digit, wrist, and forearm of the monkey with a small hand-held brush. We defined the digit region of M1 as a $2 \mathrm{~mm}$ square of cortical surface centered on the penetration site that satisfied at least one of the following criteria: (1) digit movements were obtained at a current lower than $10 \mu \mathrm{A}$; or (2) digit movements were obtained without movements of other body parts with the lowest threshold current, and the current was $<20 \mu \mathrm{A}$. Ibotenic acid (Sigma-Aldrich; $10 \mu \mathrm{g} / \mu \mathrm{l}$ in $0.1 \mathrm{M}$ PBS, pH $7.4 ; 15 \mu \mathrm{l}$ ) was then injected intracortically to destroy the digit region of M1 contralateral to the preferred hand. Multiple injections were used to spread ibotenic acid throughout the digit region, with $1.5 \mu$ linjected at a rate of $0.75 \mu \mathrm{l}$ per minute at each injection site (10 injection sites for each monkey). The location of the lesion was confirmed by the adjacent sections Nissl stained with cresyl violet (Fig. $1 C-H)$. The lesion area was defined as the area of the injection site showing gliosis and loss of neurons, as in our previous study (Murata et 
al., 2015b). The lesion areas were visualized using an Olympus BX63 microscope and their unbiased volumes were calculated on the basis of Cavalieri's principle (for review, see Mayhew, 1992) using StereoInvestigator imaging software (MBF Bioscience).

Anterograde tracer injection. More than 3 months after M1 lesioning, the anterograde neural tracer, $\mathrm{BDA}$, was injected into the ip-PMv region to assess long-range rewiring in the lesioned group. The location of the ip-PMv was determined with magnetic resonance imaging (MRI) and visual inspection of the sulcal landmarks based on the Saleem and Logothetis anatomical atlas (Saleem and Logothetis, 2007). We did not perform ICMS of PMv for several reasons. First, our previous electrophysiological study suggested that motor recovery in M1-lesioned animals does not involve the direct action of the local PMv neurons onto the lower motor neurons for hand movements (Murata et al., 2015b). This is based on the finding that ICMS of the ipsilesional PMv (with an amplitude of $<100 \mu \mathrm{A}$ ) did not elicit any hand movement. Second, in our investigations, ICMS up to $100 \mu \mathrm{A}$ elicited hand movement in very limited penetrations even in the intact PMv, and the ICMS did not elicited orofacial movements in any penetration in the PMv of some intact macaques. Third, our previous neuroimaging study suggested that an area of the PMv where ICMS elicited hand movements before lesion induction plus its rostrolateral area has a pivotal role in the functional recovery of M1-lesioned animals, because local cerebral blood flow was increased in this area during hand motor execution after recovery. This increased flow was functional since pharmacological inactivation of this area disrupted the recovered hand movement (Murata et al., 2015b). In the present study, we aimed to inject BDA into the ventral bank of both the arcuate spur and the genu of the arcuate sulcus, which have been considered as the hand area of the PMv (PMv-h) from both anatomical and electrophysiological observations (Dum and Strick, 1991; Godschalk et al., 1995; Stark et al., 2007; Murata et al., 2015b; Dea et al., 2016; Higo et al., 2016; Quessy et al., 2016; Fig. 2H-L). In each animal, we calculated the unbiased volumes of the PMv-h as well as the PMv areas outside it, including a subregion where ICMS elicited orofacial movements in a previous study (Godschalk et al., 1995), based on Cavalieri's principle.

MRI was conducted with a 3T MRI scanner (Siemens MAGNETOME Allegra) and a customized multi-array 4-channel coil (Takashima Seisakusho). The scanning protocol was as follows: three-dimensional magnetization-prepared rapid acquisition with gradient echo sequence, repetition time $=2500 \mathrm{~ms}$, echo time $=4.73 \mathrm{~ms}$, inversion time $=1030 \mathrm{~ms}$, isotropic voxel size $=0.5 \mathrm{~mm}$, matrix $=256 \times 256 \times$ 224 with four averages. Animals in the intact group also underwent MRI and were injected with BDA in the ip-PMv. MR images were manually registered to the stereotaxic space of each monkey, and the location of the ip-PMv was identified and recorded as a coordinate of the stereotaxic space.

One-half microliter (Monkeys A, B, D-F) or $1 \mu \mathrm{l}$ (Monkey C) of $10 \%$ BDA in $0.01 \mathrm{~m}$ phosphate buffer (PB; Sigma-Aldrich), $\mathrm{pH} 7.4$, was injected at nine sites ( 3 tracks $\times 3$ depths; track interval: $2 \mathrm{~mm}$ ) in the ip-PMv with a Hamilton microsyringe under deep anesthesia using 3\% isoflurane. The stereotaxic coordinates of BDA injection tracks and the precise period from BDA injection to perfusion are presented in Table 1. BDA injection in the ip-PMv was confirmed by the adjacent sections with immunohistochemistry (see Results; Fig. 2). The dense core of BDA injections was defined as the area of the injection site where cells and terminals could not be easily differentiated, as in a previous study (Dancause et al., 2005). The unbiased volumes of the BDA injection core were calculated based on Cavalieri's principle, as described.

One month after BDA injections, animals were killed with overdose pentobarbital $(100 \mathrm{mg} / \mathrm{kg})$, the brain was removed, and the brain tissues were prepared for histological analysis using methods previously described (Yamamoto et al., 2013). Briefly, the macaques were perfused via the left cardiac ventricle with $4 \%$ paraformaldehyde (PFA) and $0.1 \%$ glutaraldehyde in $\mathrm{PB}, \mathrm{pH}$ 7.4. After perfusion, the brains were immediately removed and dissected into $5 \mathrm{~mm}$ tissue blocks. The cerebrum, diencephalon, and upper brainstem, including the midbrain and part of the pons, were blocked in the coronal plane. The lower brainstem, including the medulla oblongata and most of the pons and cerebellum, was blocked in the transverse ( 3 intact and 2 lesioned monkeys, Monkeys A-E) or sagittal plane (1 lesioned monkey, Monkey F). The brain blocks were immersed in a post-fixative solution containing 2\% PFA and 5\% sucrose in $\mathrm{PB}$, infiltrated with sucrose, frozen in a dry-ice/acetone bath, and then stored at $-80^{\circ} \mathrm{C}$ until dissection.

Histological preparation for anterograde tracing. The brain slices (16 $\mu \mathrm{m}$ thick) were processed with the Neuronal Tracer Kit according to the manufacturer's manual (BDA-10,000; Neuronal Tracer Kit N-7167, Invitrogen). Briefly, after several rinses in PBS, the sections were treated for endogenous peroxidase with $0.3 \% \mathrm{H}_{2} \mathrm{O}_{2}$ in methanol for $30 \mathrm{~min}$ to diminish the background. The sections were rinsed in PBS and incubated in PBS with $0.2 \mu \mathrm{g} / \mathrm{ml}$ avidin-horseradish peroxidase (HRP) conjugate reagent for $3 \mathrm{~h}$. After washing with PBS and Tris-buffer, the sections were dipped in peroxidase substrate solution with $0.5 \mathrm{mg} / \mathrm{ml} \mathrm{DAB}$ and $0.3 \%$ nickel ammonium in Tris-buffer, $\mathrm{pH}$ 7.6, for $1 \mathrm{~h}$, followed by peroxidase substrate solution containing $\mathrm{H}_{2} \mathrm{O}_{2}$ for $10 \mathrm{~min}$. The sections were dehydrated through serial washes in $70,80,90$, and $100 \%$ ethanol, washed in xylene three times for 5 min each, and coverslipped with Permount histological mounting medium (Fisher Scientific). To minimize intrinsic variability between experiments, sections from as many areas as possible were stained simultaneously.

Immunohistochemistry. Immunofluorescence was performed to confirm the synaptic connection between the ip-PMv and ip-FN by using antibodies against the presynaptic vesicle protein (Table 2): VGLUT1 (Millipore, MAB5502; Mouse; 1:2000) and synaptophysin (Millipore, MAB329; Mouse; 1:4000; Cai et al., 2010).

The sections were subjected to heat-mediated antigen retrieval with citric acid, $\mathrm{pH}$ 6.0, and then treated for endogenous peroxidase with $0.3 \% \mathrm{H}_{2} \mathrm{O}_{2}$ in methanol for $30 \mathrm{~min}$. After treatment with normal serum blocking solution for $1 \mathrm{~h}$, the sections were amplified with a Tyramide Signal Amplification Biotin System (PerkinElmer), and incubated for $48 \mathrm{~h}$ at $4^{\circ} \mathrm{C}$ with both the Streptavidin AlexaFlour 546 IgG (Invitrogen; $1: 100)$ and the primary antibodies against the presynaptic vesicle protein (VGLUT1 and synaptophysin) diluted in PBS containing 5\% BSA and $0.02 \%$ sodium azide. After washing with PBS, sections were incubated for $120 \mathrm{~min}$ at room temperature with the secondary antibody, AlexaFluor 488 goat anti-mouse IgG $(\mathrm{H}+\mathrm{L})$ conjugate (Invitrogen; mouse, 1:400), and then stained with $4^{\prime}, 6^{\prime}$-diamidino-2-phenylindole hydrochloride (DAPI; Dojindo; $5 \mu \mathrm{g} / \mathrm{ml}$ ) for $5 \mathrm{~min}$ to identify cells. The sections were washed with PBS and mounted with Fluoromount/Plus (Diagnostic BioSystems). In addition, we confirmed secondary antibody specificity by using sections incubated without primary antibody and finding no specific signals (data not shown).

Enzyme-immunohistochemistry was performed to characterize cell types to which ip-PMv neurons projected by using cerebellar compartmentalization markers (Table 2): Aldoc (Santa Cruz Biotechnology, sc271593; mouse, 1:2000) and PLCB3 (Santa Cruz Biotechnology, sc133231; mouse1:2000). These molecules are known to segregate cerebellar cortex into a series of parasagittal stripes, as well as cerebellar nuclei into multiple compartments (Brochu et al., 1990; Sugihara and Shinoda, 2004; Sarna et al., 2006; for review, see Apps and Hawkes, 2009; Consalez and Hawkes, 2013). After treatment with $0.3 \% \mathrm{H}_{2} \mathrm{O}_{2}$ in methanol and several rinses in PBS, the sections were treated for 10 min with blocking solution (BLOXALL Blocking Solution, Vector Laboratories) to inactivate endogenous peroxidases, pseudo-peroxidases, and alkaline phosphatase. After treatment with normal serum blocking solution for 30 min, the sections were incubated for $48 \mathrm{~h}$ at $4^{\circ} \mathrm{C}$ with the primary monoclonal antibodies diluted in PBS containing 5\% BSA and $0.02 \%$ sodium azide.

After washing, sections were incubated with ImmPRESS-AP antimouse IgG polymer detection reagent (alkaline phosphatase; MP-5402, Vector Laboratories) for $30 \mathrm{~min}$ at room temperature, and then incubated in color-developing buffer containing $p$-nitro blue tetrazolium and 5-bromo-4-chloro-3-indoyl phosphate for $0.5-1 \mathrm{~h}$ (Aldoc) or 1-2 h (PLCB3). After washing, the sections were immersed in 4\% PFA, and dehydrated through serial washes in $70,80,90$, and $100 \%$ ethanol, washed in xylene three times for $5 \mathrm{~min}$ each, and coverslipped with Permount histological mounting medium. We observed the immunore- 

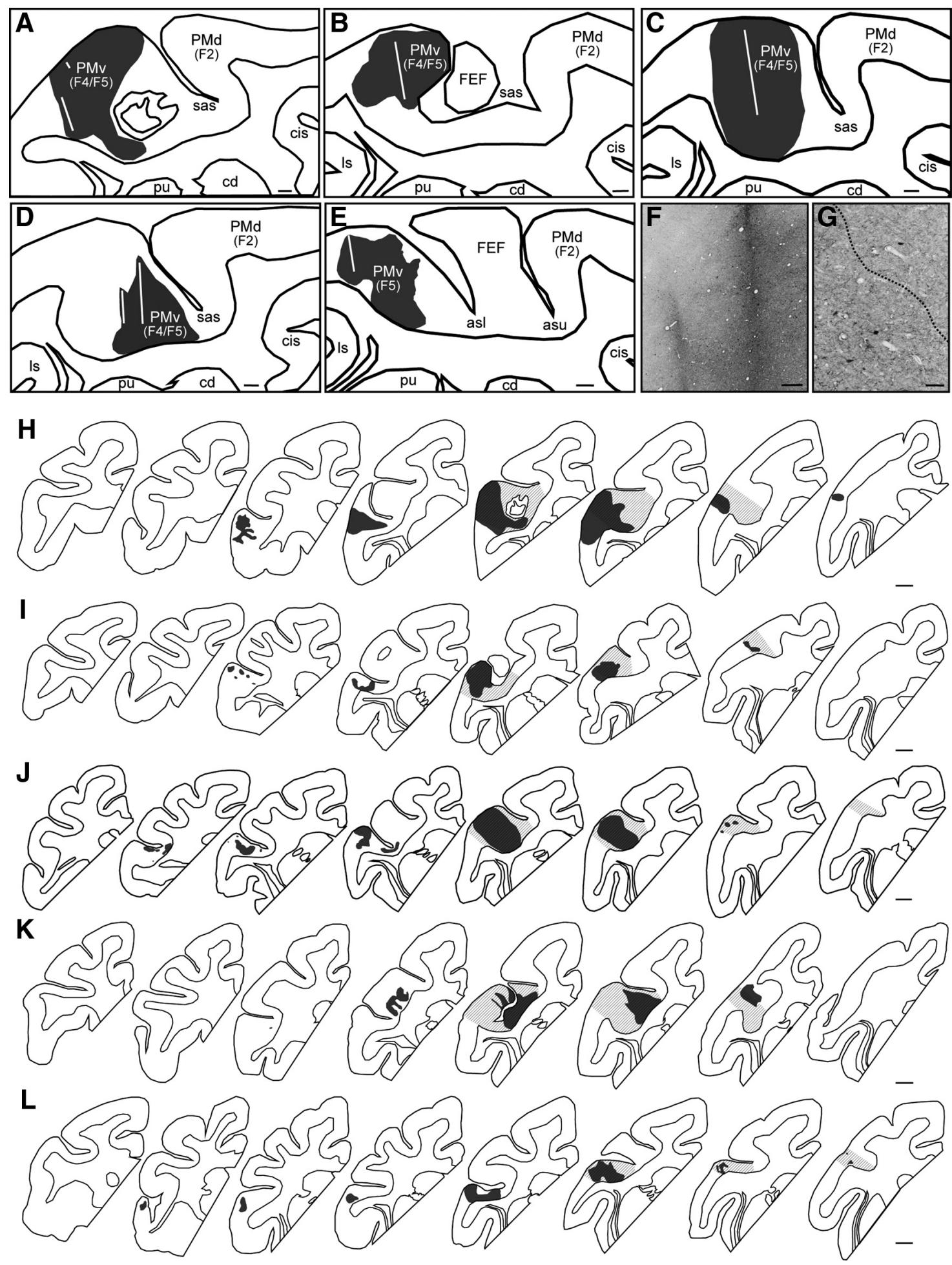

Figure 2. BDA injection to the PMv of intact and lesioned monkeys. $\boldsymbol{A}-\boldsymbol{E}$, Line drawing of the premotor area in intact $(\boldsymbol{A}$, Monkey $\mathrm{A} ; \boldsymbol{B}$, Monkey $\boldsymbol{B} ; \boldsymbol{C}$, Monkey $\boldsymbol{C})$ and lesioned monkeys (D, Monkey $D ; E$, Monkey F). This section in Monkey E was subsequently excluded because the tissue was destroyed when the adhering dura mater was removed. The core of the BDA injection is represented in black. The white line indicates the trace of the injection. $\boldsymbol{F}, \boldsymbol{G}$, High-magnification photomicrographs in Monkey D showing the trace of the injection $(\boldsymbol{F})$ and the border between the core and the halo of the BDA injection ( $\boldsymbol{G}$, dotted line). $\boldsymbol{H}-\boldsymbol{L}$, Reconstructions of serial sections including the core of the BDA injection (black) and the anatomically defined PMv hand area (gray oblique lines) in each intact ( $\boldsymbol{H}$, Monkey $A ; \boldsymbol{I}$, Monkey $B ; \boldsymbol{J}$, Monkey $C$ ) and lesioned monkey ( $\boldsymbol{K}$, Monkey $\mathrm{D} ; \boldsymbol{L}$, Monkey F), separated by $\sim 1.3 \mathrm{~mm}$ and arranged from rostral (left) to caudal (right). PMd, Dorsal premotor cortex; $F 2 / F 4 / F 5$, agranular frontal area $F 2 / F 4 / F 5$; cd, caudate nucleus; pu, putamen; sas, spur of the arcuate sulcus; ls, lateral sulcus; cis-, cingulate sulcus; asl/asu, arcuate sulcus lower/upper limb. Scale bars: $\boldsymbol{A}-\boldsymbol{E}, 1 \mathrm{~mm} ; \boldsymbol{F}, 300 \mu \mathrm{m} ; \boldsymbol{G}, 100 \mu \mathrm{m} ; \boldsymbol{H}-\boldsymbol{L}, 1 \mathrm{~mm}$.

active stripes of Aldoc and PLCB3 in the cerebellar vermis (data not shown). In addition, we confirmed secondary antibody specificity by using sections incubated without primary antibody and no specific signal was observed (data not shown).
Image acquisition and tracing. The stained slices were observed and recorded with a Keyence BZ-8000 microscope (Keyence). Image editing was performed in Adobe Photoshop CC, which included cropping, resizing, adjusting brightness and contrast, and removing obvious contaminations. 
Table 2. Antibodies used in the present study

\begin{tabular}{lllll}
\hline Marker & Antigen & Manufacturer & Species & Dilution \\
\hline \multirow{2}{*}{ Synapse } & VGLUT1 & Millipore & Mouse & $1: 2000$ \\
& Synaptophysin & Millipore & Mouse & $1: 4000$ \\
Cerebellar & Aldoc & Santa Cruz Biotechnology & Mouse & $1: 2000$ \\
& PLCB3 & Santa Cruz Biotechnology & Mouse & $1: 2000$ \\
\hline
\end{tabular}

BDA stained sections were first scanned with a light microscope at $20 \times$, to draw the contour of the section. Afterward, the same sections were systematically captured at $100 \times$ to trace the BDA-positive axons with Adobe Illustrator CC. Each brain structure was identified with the Nissl-stained sections according to an anatomical atlas (Madigan and Carpenter, 1971; Saleem and Logothetis, 2007) and the BrainMaps website (http://brainmaps.org/).

Experimental design and statistical analysis. The numbers of BDAlabeled axons and boutons in the deep cerebellar nucleus were counted visually by an experimenter (N.H.) who was blinded to the section identity. Three sites in each cerebellar nucleus (rostral, medial, and caudal) in the transverse sections (Monkeys A-E) and two sites in each cerebellar nucleus (lateral and medial) in the sagittal sections (Monkey F) were analyzed for quantification of axons and boutons. To normalize tracer uptake efficacy across animals, the number of BDA-positive fibers in the cerebellar nucleus was scaled by the total number of BDA-positive fibers in the corticospinal tract at the cerebral peduncle in each animal. The chosen section of the cerebral peduncle included both the magnocellular part of the red nucleus and the lateral terminal nucleus of the accessory optic tract (Table 1; Ishida et al., 2016). The normalized values were expressed as the mean number of BDA-labeled axons or boutons in the two or three sites of each cerebellar nucleus divided by the number of fibers in the cerebral peduncle for each animal. Using the corticospinal tracts for the normalization procedure is reasonable because: (1) PMv contains neurons giving rise to corticospinal tracts (Borra et al., 2010); (2) structural changes of corticospinal tract are not known to occur in the brain regions above the cerebral peduncle, but occur at the level of the spinal cord to compensate the function of the damaged CNS; and (3) the number of BDA-positive fibers in the cerebral peduncle was most likely associated with the amount of injected BDA (Monkey C, in which we injected double the amount of BDA, showed the largest number of BDA-positive fibers among the 6 monkeys examined).

Statistical analyses were performed using Prism v 6.02 (GraphPad Software). The Mann-Whitney $U$ test was used to quantitatively compare the boutons/axons in the cerebellar nuclei between intact (Monkeys A-C) and lesioned monkeys (Monkeys D-F). The Kruskal-Wallis test with Dunn's multiple-comparisons test were also used for quantitative analysis of boutons in the cerebellar nuclei (fastigial, interposed, and dentate) of intact monkeys (Monkeys A-C). $P$ values $<0.05$ were used to refute the null hypothesis.

In the colocalization analysis of immunofluorescence signals, we calculated the rate of colocalization of BDA-positive boutons with the synaptic markers in the ip-FN of lesioned monkeys (Monkeys D-F). One section stained with VGLUT1/BDA or synaptophysin/BDA was captured with the Keyence BZ-8000 microscope (Keyence) at $600 \times$ from each monkey. The numbers of synaptic markers as well as BDA-positive boutons were visually counted as described above for the bright-field quantification in a $100 \times 100 \mu \mathrm{m}$ square on the captured image. One square, where BDA-positive axons were clearly visible, was analyzed in each monkey. For both BDA-positive boutons and the synaptic markers, we defined signals as positive when they showed a signal intensity $>80 \%$ of the maximum gray level intensity within the square.

\section{Results}

Functional recovery of hand movements after M1 lesion

Before the lesion, all monkeys were well trained to perform the precision grip task and success rates reached $100 \%$ as shown in Figure $1 B$. Ibotenic acid injection in the M1 hand digit area resulted in a focal lesion in which there was loss of neurons and gliosis (Fig. $1 \mathrm{C}-\mathrm{H}$ ). The average estimated volume of the lesion area was $55.4 \pm 0.9 \mathrm{~mm}^{3} \pm \mathrm{SE}$ (Table 1 ). Immediately after the induction of the M1 lesion (Fig. $1 B$, arrowhead), the three lesioned monkeys could not perform the precision grip because of hand paralysis. Functional recovery of hand movements was observed during the course of daily post-lesion training on the precision grip task. The mean success rate gradually increased after the M1 lesion and reached a plateau level $>90 \%, 2$ months postlesion (Monkey D: $97 \%$ at 57 d, $100 \%$ at 64 d; Monkey E: $96.7 \%$ at $56 \mathrm{~d}, 100 \%$ at $63 \mathrm{~d}$; Monkey F: $90.3 \%$ at $55 \mathrm{~d}, 93.3 \%$ at $62 \mathrm{~d}$, $93.3 \%$ at $69 \mathrm{~d}, 100 \%$ at $83 \mathrm{~d})$. The recovery pattern resembled that in our previous studies (Murata et al., 2008, 2015b).

\section{Sites of BDA injection in ip-PMv}

Figure 2 shows the sections of BDA injection sites for all animals, except for a lesioned animal (Monkey E) that was removed from the analysis because the tissue including the ip-PMv was unexpectedly destroyed when removing the adhering dura mater. We confirmed that BDA was successfully injected into the ventral bank of both the arcuate spur and the genu of the arcuate sulcus (PMv-h), as planned (see Materials and Methods), in all monkeys, including Monkey E. However, most of the brain sections of Monkey E could not be assessed because of dura adhesion and tissue damage. Moreover, most of the BDA injection cores were observed within the PMv (F4/F5) of the monkeys examined (Fig. 2 ). The average estimated volumes $( \pm S E)$ of the BDA injection core in the intact and lesion monkeys were 107.4 \pm 28.3 and $62.2 \pm 13.3 \mathrm{~mm}^{3} \pm \mathrm{SE}$, respectively (Table 1 ). Within the $\mathrm{PMv}-\mathrm{h}$, the average volume percentages of the BDA injection core were $45.7 \pm 4.2 \%$ (mean $\pm \mathrm{SE}$ ) of the total PMv-h in the intact (the percentages in Monkeys A-C were 45.4, 38.6, and $53.0 \%$, respectively) and $32.4 \pm 3.2 \%$ (the percentages in Monkeys $\mathrm{D}$ and $\mathrm{F}$ were 29.2 and $35.6 \%$, respectively) in the monkeys with lesions. The BDA cores outside the PMv-h were relatively small: $15.5 \pm 7.5 \%(30.5,6.9$, and 9.1\% in Monkeys A-C, respectively) and $6.9 \pm 6.7 \%$ (0.2 and 13.5\% in Monkeys D and F, respectively) in intact and lesioned monkeys, respectively. In the entire BDA injection core, the average volume percentages of the BDA injection core within the PMv-h were 71.5 $\pm 7.1 \%$ (mean \pm SE; the percentages in Monkeys A-C were 57.3, 78.4, and 78.6\%, respectively) and $62.2 \pm 14.9 \%$ (the percentages in Monkeys D and $\mathrm{F}$ were 77.1 and $47.3 \%$, respectively) in intact and lesioned monkeys, respectively.

\section{Direct cortico-cerebellum projections from the ip-PMv}

BDA-labeled axon terminals in both the lesioned and intact monkeys were identified in several brain regions including the medial reticular nucleus, raphe nucleus, inferior olivary complex, and cerebral peduncle (Fig. 3A-F). In intact monkeys, the terminals in the medial reticular nucleus were more abundant than that in the raphe nucleus or inferior olivary complex, in line with previous studies (Borra et al., 2010; Fregosi et al., 2017). There were a lower number of terminals in the medial reticular nucleus of the M1-lesioned monkeys than in the intact monkeys, which is also consistent with previous findings (Fregosi et al., 2018).

Among the transverse sections containing the lower brainstem and cerebellum, a marked difference was observed between the groups in the deep cerebellar nuclei. BDA-labeled terminals were observed in the deep cerebellar nuclei of M1-lesioned animals, whereas there were few terminals in the intact animals. These differences were also seen in the external cuneate nucleus. High-magnification photomicrograph revealed BDA-positive axons and boutons in the cerebellar nucleus of a lesioned monkey (Fig. 3G,H). We quantified the numbers of BDA-labeled boutons 

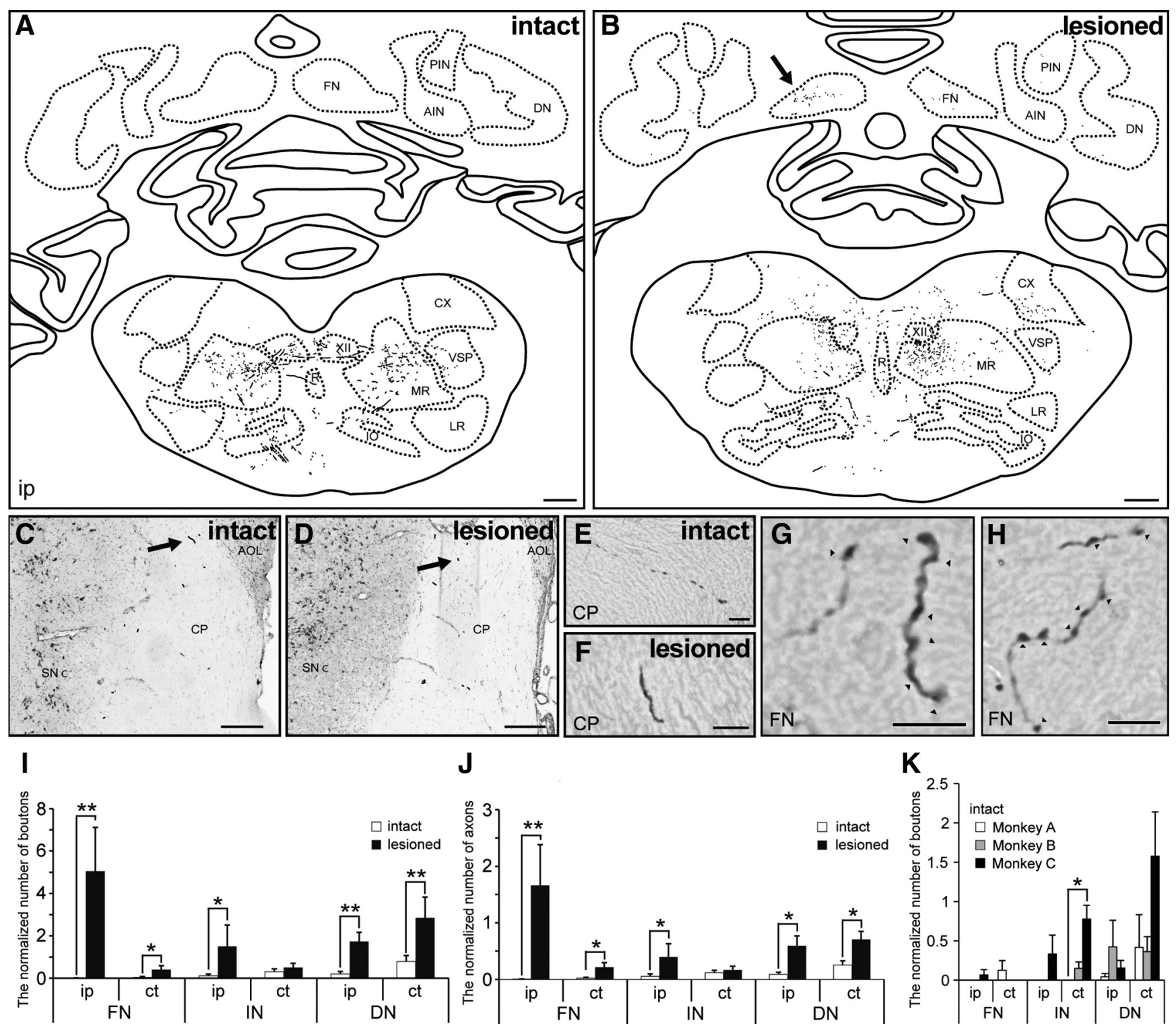

Figure 3. Comparison of PMv projections to cerebellar nuclei between intact and lesioned monkeys. $A, B$, Reconstructions of a transverse section including brainstem and cerebellar nuclei of an intact $(\boldsymbol{A}$, Monkey $A)$ and lesioned monkey ( $\boldsymbol{B}$, Monkey D). $\boldsymbol{C}-\boldsymbol{F}$, The BDA-positive axons running through the cerebral peduncle of intact $(\boldsymbol{C}, \boldsymbol{E}$, Monkey $B)$ and lesioned monkeys $(\boldsymbol{D}, \boldsymbol{F}$, Monkey $\mathrm{E})$. The arrows in $\boldsymbol{C}$ and $\boldsymbol{D}$ indicate the BDA-positive axon in $\boldsymbol{E}$ and $\boldsymbol{F}$, respectively. $\boldsymbol{G}, \boldsymbol{H}$, High-magnification photomicrograph showing the BDA-positive axons and boutons in the fastigial nucleus of Monkey $D(B$, arrow). The arrowheads indicate the BDA-positive boutons. $\boldsymbol{I}, \boldsymbol{J}$, Quantitative analysis for boutons $(\boldsymbol{I})$ and axons $(\boldsymbol{J})$ in the cerebellar nuclei (fastigial, interposed, and dentate) of intact and lesioned monkeys. ${ }^{*} p<0.05,{ }^{* *} p<0.01$, according to a Mann-Whitney $\boldsymbol{U}$ test. $\boldsymbol{K}$, Quantitative analysis of boutons in the cerebellar nuclei (fastigial, interposed, and dentate) of intact monkeys (Monkeys A-C). ${ }^{*} p<0.05$, according to a Kruskal-Wallis test with Dunn's multiple-comparisons test. The number of BDA-positive boutons and axons was normalized by the number of labeled fibers at the cerebral peduncle level. Values are shown as mean \pm SE. ct, Contralesional side; A/PIN, anterior/posterior interposed nucleus; DN, dentate nucleus; XII, hypoglossal nucleus; M/LR, medial/lateral reticular nucleus; $C X$, external cuneate nucleus; $R$, raphe nucleus; VSP, spinal trigeminal nucleus; 10 , inferior olivary complex; $C P$, cerebral peduncle; $S N c$, pars compacta of substantia nigra; $A 0 \mathrm{~L}$, lateral terminal nucleus of accessory optic tract. Scale bars: $\boldsymbol{A}, \boldsymbol{B}, 1 \mathrm{~mm} ; \boldsymbol{C}, \boldsymbol{D}, 300 \mu \mathrm{m} ; \boldsymbol{E}-\boldsymbol{H}, 10 \mu \mathrm{m}$.

and axons in each deep cerebellar nucleus (fastigial, interposed, and dentate), and found that the lesioned group showed higher numbers of BDA-labeled boutons and axons in the ipsilesional deep cerebellar nucleus compared with the intact group (Fig. $3 I, J)$. This increase was particularly evident in the ip-FN (the normalized number of boutons $=5.046 \pm 2.068$ vs $0.022 \pm$ 0.022 , lesion vs intact $\pm \mathrm{SE}, U=10, p=0.0041$; that of axons $=$ $1.662 \pm 0.721$ vs $0.007 \pm 0.007 ; U=10, p=0.0041$; MannWhitney $U$ test, two-tailed). Despite injecting double the amount of BDA into Monkey C (Table 1), the number of boutons was low in the ip-FN of this monkey (Fig. $3 \mathrm{~K}$; the normalized number of boutons $=0.066 \pm 0.066$ ).
Serial transverse sections of the ip-FN showed that BDAlabeled axon terminals were mainly observed in the central part in both the medial-lateral and ventral-dorsal axes (Fig. 4).

Moreover, serial sagittal sections showed that BDA-labeled terminals were mainly located in the middle part of the ip-FN region in the rostral-caudal axis (Fig. $5 B, H$ ). The terminals were also observed in the lobule Vf of cerebellar cortex (Fig. $5 D, I$ ).

Some of the BDA-positive boutons in the ip-FN were colocalized with synaptic markers for VGLUT1, as well as with synaptophysin, in all lesioned monkeys examined (Fig. 6). The average percentage of BDA-positive boutons with VGLUT1 of the total BDA-positive boutons counted was $84.4 \pm 3.9 \%$ [mean $\pm \mathrm{SE}$; the 


\section{Monkey D}
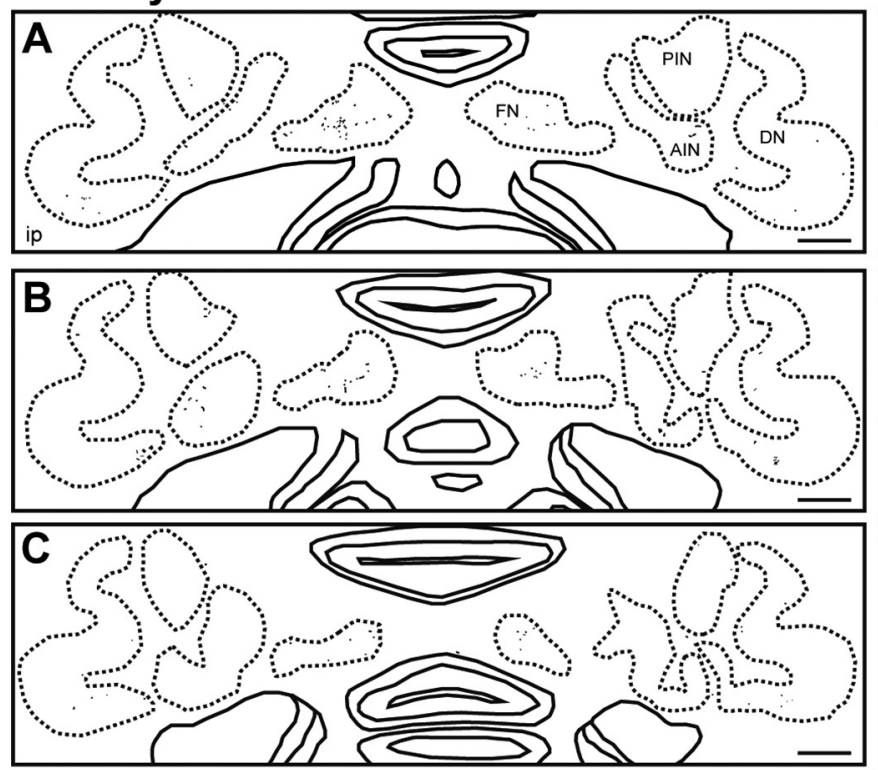

Monkey E
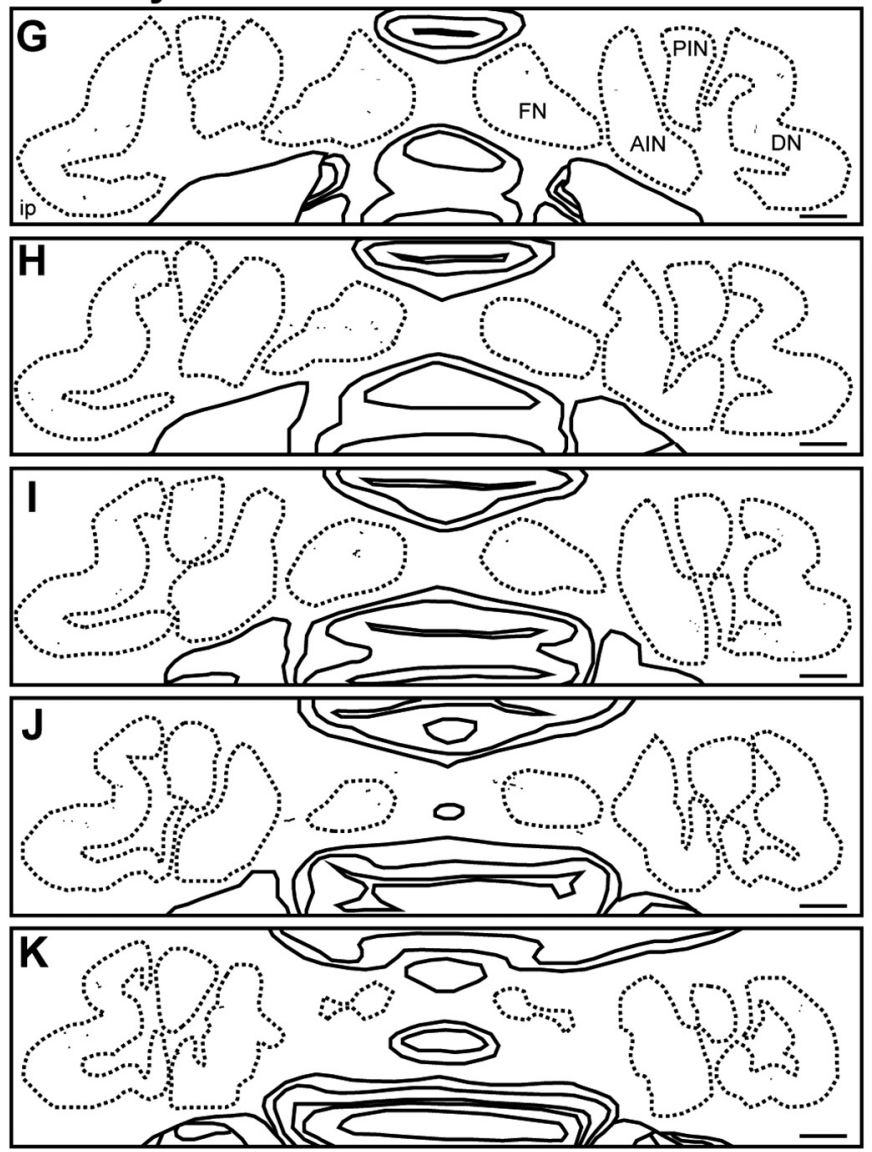
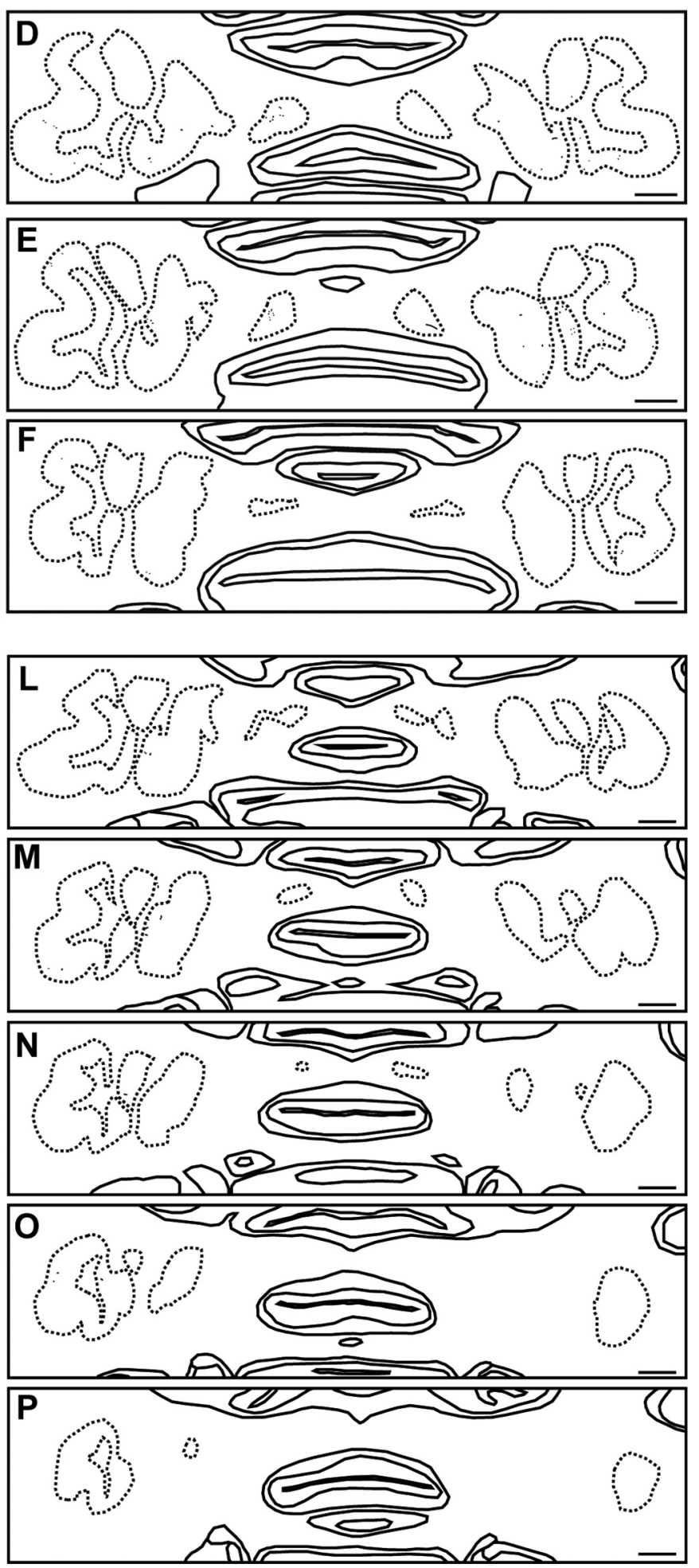

Figure 4. ip-PMv projections to cerebellar nuclei in lesioned Monkeys $D$ and $E$. Reconstructions of serial transverse sections including cerebellar nuclei of the lesioned monkeys ( $\boldsymbol{A}-\boldsymbol{F}$ : Monkey $D$, $\boldsymbol{G}-\boldsymbol{P}$ : Monkey E), separated by $160 \mu \mathrm{m}$ and arranged from rostral $(\boldsymbol{A}, \boldsymbol{G})$ to caudal $(\boldsymbol{F}, \boldsymbol{P})$. A/PIN, Anterior/posterior interposed nucleus; DN, dentate nucleus. Scale bars: $\boldsymbol{A}-\boldsymbol{P}, 1 \mathrm{~mm}$.

percentages (numbers) in Monkeys D-F were 86.4\% (19/22), $76.9 \%(10 / 13)$, and $90.0 \%(9 / 10)$, respectively] and for synaptophysin were $82.1 \pm 8.9 \%$ [72.7\% (8/11), 73.7\% (14/19), and $100 \%$ (9/9) in Monkeys D-F, respectively]. These findings indicate that the axon terminals from ip-PMv projections form functional synaptic connections to ip-FN neurons.
Overall distribution of cerebellar compartmentalization markers, Aldoc and PLCB3, was not much different between the FN region of intact animals (Fig. $7 A, C, E$ ) and that of lesioned animals (Fig. $7 B, D, F$ ). Prominent immunoreactivity of Aldoc was observed in the $\mathrm{FN}$, but was weaker in the dorsal than in the ventral portion (Fig. 7C, area surrounded by black dotted line, 
$D, H$, yellow dotted line). The prominent expression of PLCB3 was distributed across both Aldoc-positive and Aldoc-negative portions (Fig. 7E, area surrounded by black solid line, $F, H$, red solid line).

Comparison of the spatial distribution of BDA labeling with those of the compartment markers in lesioned monkeys confirmed that the terminals of BDAlabeled axons were distributed in the Aldoc-negative portion of the ip-FN region (Fig. $7 D, G, H$ ), which is thought to be part of the somatosensory system of the FN (for review, see Sugihara, 2011). Among the compartments characterized by the expression of PLCB3, the location of ip-PMv axon terminals was observed in the PLCB3-positive zone (Fig. $7 F-H$ ). However, the functional significance of this marker is yet to be addressed.

\section{Discussion}

In the present study, we provide evidence that neuroanatomical reorganization occurs in the subcortical projection from the ip-PMv to deep cerebellar nuclei following lesioning of the M1 digit area. These projections to the deep cerebellar nuclei were remarkably altered in the middle of the ip-FN region in which we confirmed no or weak expression of Aldoc. Formation of functional synapses between ip$\mathrm{PMv}$ projections and ip-FN neurons were confirmed with multi-fluorescence staining of synaptic markers.

These findings of anatomical rewiring support our previous study using the same animal model, which revealed functional connectivity of the cerebellum with a PPI analysis (see Table 3 in Murata et al., 2015b), and suggested a pivotal role for the cerebellum in recovering motor behavior. Moreover, a previous study reported that the short corticocortical projection from the ip-PMv to the area $1 / 2$ was increased in motor-recovered monkeys after lesioning of the M1 hand area compared with intact monkeys (Dancause et al., 2005). The approximate distance from the ip-PMv to area $1 / 2$ is $\sim 10 \mathrm{~mm}$, whereas that from the ip-PMv to ip-FN is $\sim 40 \mathrm{~mm}$ in adult macaque monkeys (Saleem and Logothetis, 2012). Together, our results provide the first demonstration that after M1 lesion and motor recovery the ip-PMv reorganizes neuroanatomical projections to distant subcortical regions, specifically cerebellar deep nuclei. These findings indicate that even a small amount of localized damage to the adult brain can trigger large-scale reorganization of neural circuits across diverse parts of the CNS.
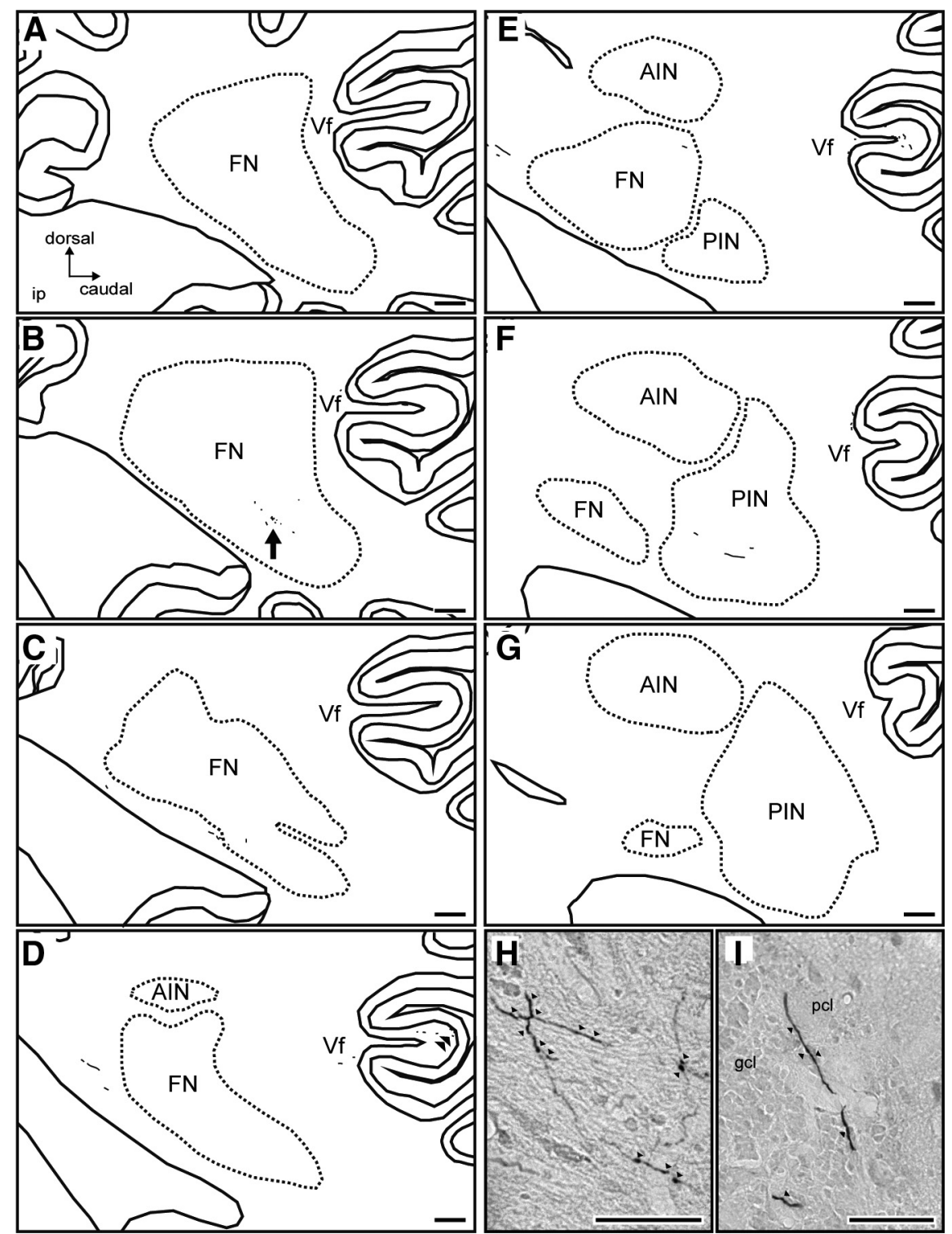

Figure 5. ip-FN projections to cerebellar nuclei in lesioned Monkey $F$. Reconstructions of serial sagittal sections including the ip-FN of lesioned Monkey F, separated by $320 \mu \mathrm{m}$ and arranged from medial $(\boldsymbol{A})$ to lateral $(\boldsymbol{G})$. $\boldsymbol{H}, \boldsymbol{I}$, High-magnification photomicrographs showing BDA-positive axons and boutons in the ip-FN $(\boldsymbol{H}$, arrow in $\boldsymbol{B})$ and in lobule Vf of the cerebellar cortex $(\boldsymbol{I}$, double arrowheads in $\boldsymbol{D})$. Arrowheads in $\boldsymbol{H}$ and $\boldsymbol{I}$ indicate the boutons of BDA-positive neurons. A/PIN, Anterior/posterior interposed nucleus; DN, dentate nucleus; Vf, lobule Vf; pcl, Purkinje cell layer; gcl, granule cell layer. Scale bars: $\boldsymbol{A}-\mathbf{G}, 500 \mu \mathrm{m} ; \boldsymbol{H}, \mathbf{I}, 50 \mu \mathrm{m}$.
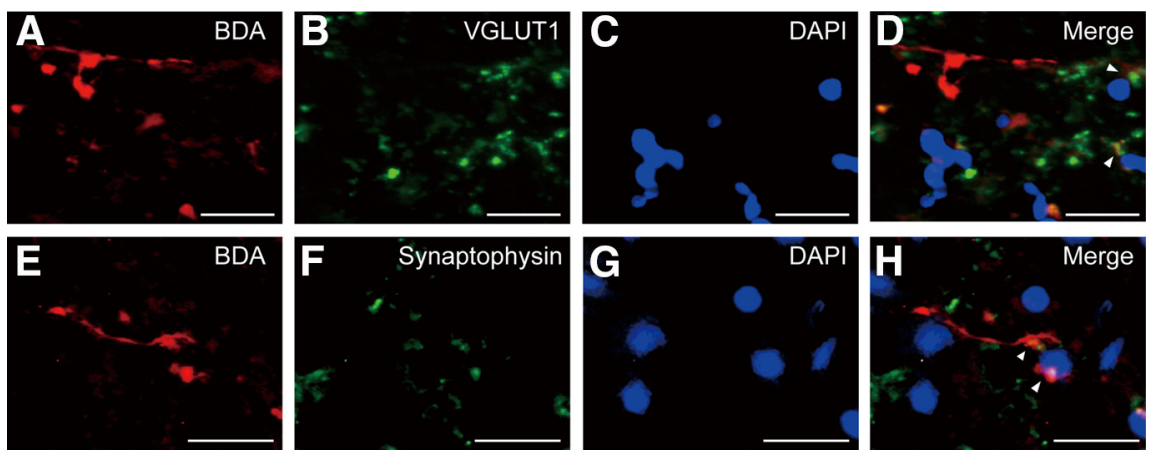

Figure 6. Functional synapses between ip-PMv projections and ip-FN neurons. $A-D$, Multi-staining study with VGLUT1 in Monkey D. $\boldsymbol{A}, \mathrm{BDA} ; \boldsymbol{B}$, VGLUT1; C, DAPI; D, merged image (BDA, red; VGLUT1, green; DAPI, blue). Arrowheads indicate consistent colocalization of BDA with VGLUT1. $\boldsymbol{E}-\boldsymbol{H}$, Multi-staining study with synaptophysin in Monkey D. $\boldsymbol{E}, \mathrm{BDA} \boldsymbol{F}$, synaptophysin; $\mathbf{G}, \mathrm{DAPI}$; $\boldsymbol{H}$, merged image (BDA, red; synaptophysin, green; DAPI, blue). Arrowheads indicate consistent colocalization of BDA with synaptophysin. Scale bars: $\boldsymbol{A}-\boldsymbol{H}, 20 \mu \mathrm{m}$. 

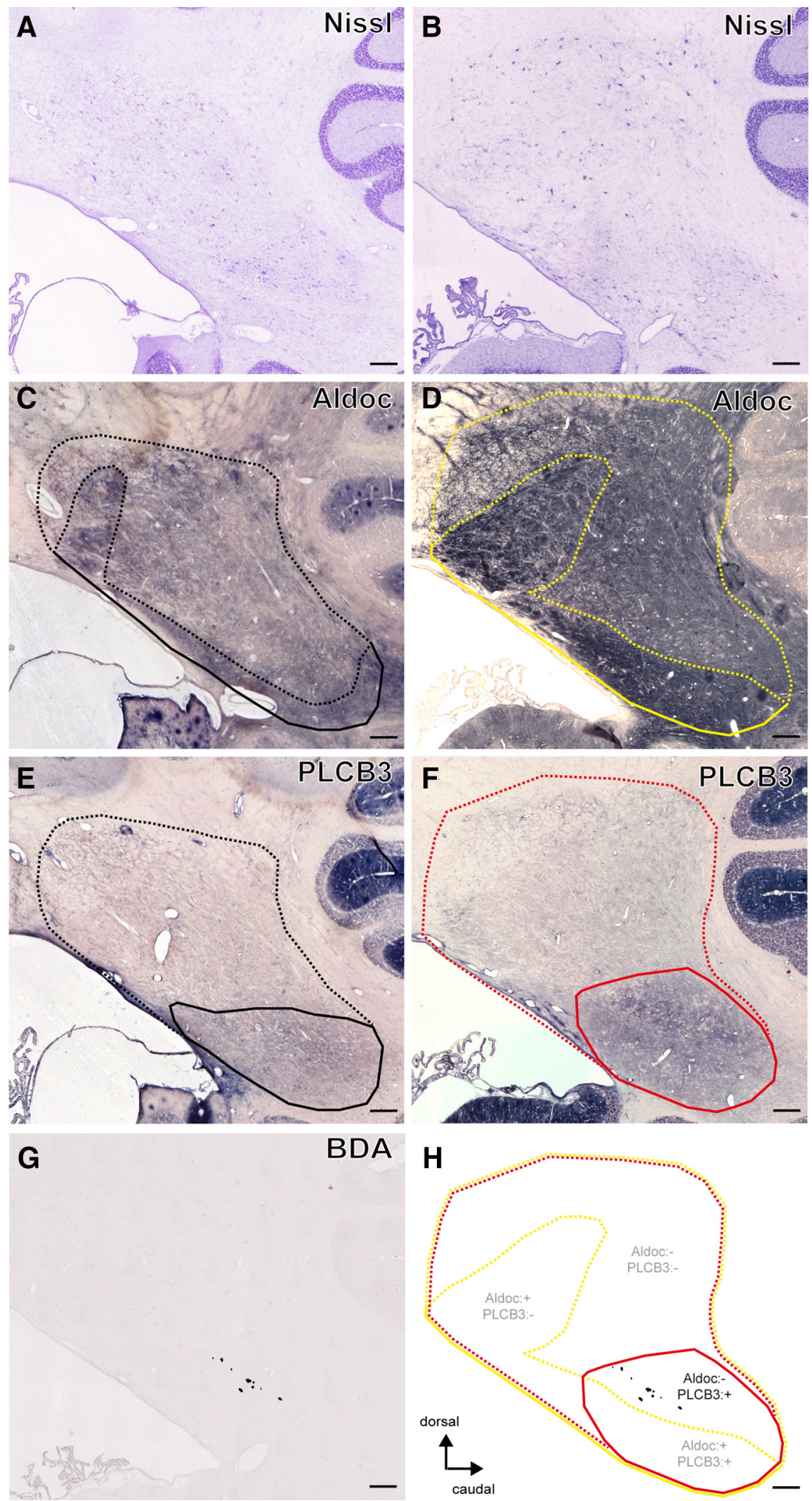

Figure 7. Characterization of ip-FN projections from the ip-PMv. Sagittal sections of the ip-FN of an intact $(A, C, E$; Monkey $G)$ and lesioned monkey ( $\boldsymbol{B}, \boldsymbol{D}, \boldsymbol{F}-\boldsymbol{H}$; Monkey F). Nissl-stained $(\boldsymbol{A}, \boldsymbol{B})$ and adjacent sections showing $\operatorname{Aldoc}(\boldsymbol{C}, \boldsymbol{D}), \operatorname{PLCB} 3(\boldsymbol{E}, \boldsymbol{F})$, and BDA labeling $(\boldsymbol{G})$. Summary montage of these sagittal sections from the lesioned monkey illustrating the heterogeneity of the FN based on differential expression of Aldoc and PLCB3 $(\boldsymbol{H})$. Areas surrounded by dotted and solid lines indicate the negative and positive zone of Aldoc (intact, black; lesioned, yellow) or PLCB3 (intact, black; lesioned, red), respectively. Tissue section $\mathbf{G}$ is the same as that shown in Figure 5B. Scale bars: $300 \mu \mathrm{m}$.
Increased subcortical projections from the ip-PMv to ip-FN after M1 lesion

We clearly observed a significant increase in projections from the ip-PMv to deep cerebellar nuclei in the lesioned monkeys, although a direct cortico-cerebellar pathway has not been reported thus far in intact monkeys. Indeed, intact monkeys showed that direct projections from the ip-PMv to deep cerebellar nuclei were scarce, although there were abundant projections to pontine nuclei, an indirect cortico-cerebellar connection (data not shown). We also observed a small amount of BDA-positive axons in the dentate nucleus, a part of the "cerebro-cerebellum", which is thought to be involved in motor programming (Kandel et al., 2012). These axons were most frequently observed in Monkey $\mathrm{C}$ of the intact group, and the youngest monkey (Table 1). It is well known that elimination of long-range axon collaterals occurs during the refinement of subcortical targets arising from cortical pyramidal neurons (Stanfield et al., 1982; Stanfield and O'Leary, 1985a,b). Together, we suggest that, while the direct cortico-cerebellar pathway may be mostly pruned away during development, a small portion of the pathway remains in adults, which may be reorganized to compensate for functional networks and behaviors. That is, rather than de novo regeneration of whole long-distance axons, local sprouting may occur from the existing axons that terminate in the ip-FN region.

It is of note that, among the deep cerebellar nuclei, the ip-FN showed the most prominent increase in BDA-positive axons descending from the ip-PMv. The FN is the phylogenetically oldest nucleus in the cerebellum, and it mainly receives somatosensory and proprioceptive inputs from the spinal cord. It is therefore considered as a key component of the "spinocerebellum” (Kandel et al., 2012). A recent anatomical study in macaques has reported that cortical motor areas, including the primary motor and medial premotor areas, provide dense inputs to the vermis, the output of which are directed largely to the FN (Coffman et al., 2011). Therefore, it is interesting that animals showing motor recovery had reorganized projections from the ip$\mathrm{PMv}$ to the ip-FN, which may potentially compensate for lost functional information usually conveyed from the primary motor cortex to cerebellar vermis, and hence to $\mathrm{FN}$.

Functional implications of remodeling The current study provides evidence of increased fronto-cerebellar projections following motor lesion and recovery. We histologically confirmed the location of 


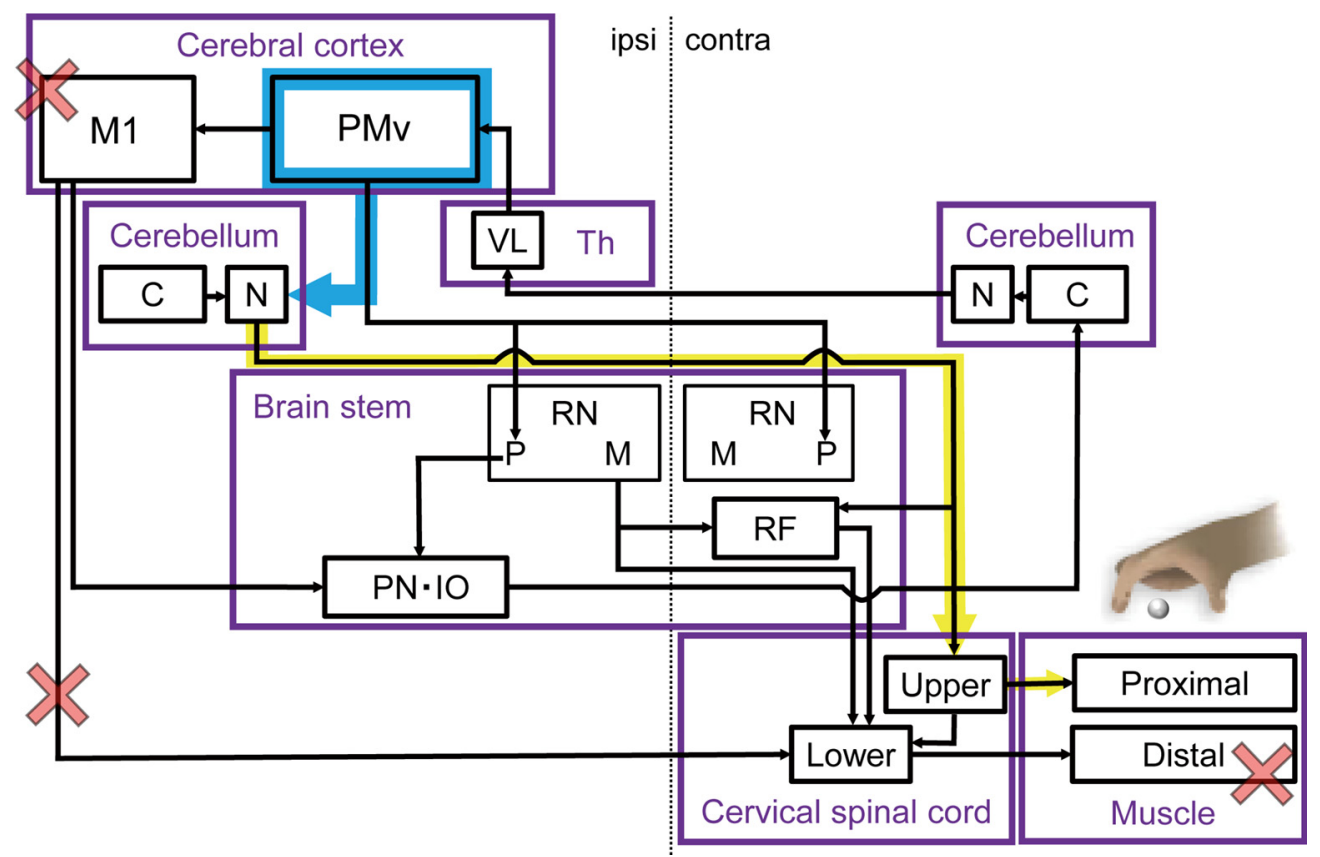

Figure 8. Conceptual diagram illustrating a hypothesis of the functional recovery mechanism based on neuroanatomical reorganization after M1-lesion induction. After induction of the M1 lesion, motor paralysis of distal hand muscles was caused by the dysfunction of corticospinal tracts projecting from $\mathrm{M} 1$ to the lower cervical spinal cord (red cross). When dexterous movements were recovered 3 months after $\mathrm{M} 1$ lesion, projections from the ip-PMv to ip-FN were strengthened (blue arrow). Reinforcement of the premotor cortical-cerebellar pathway may accompany activation of fastigial-spinal projections (yellow arrow), by which indirect cortico-spinal projections may be reorganized for functional compensation of distal muscle movement in upper extremities (via the acquisition of compensatory movement using proximal muscles). contra, Contra-lesional side; Th, thalamus; $\mathrm{VL}$, ventral lateral nucleus; $\mathrm{RN}$, red nucleus; $\mathrm{P}$, parvocellular; $\mathrm{M}$, magnocellular; $\mathrm{C}$, cortex; $\mathrm{N}$, nucleus; RF; reticular formation; PN, pontine nucleus; 10 , inferior olivary nucleus.

BDA in the PMv but did not perform ICMS of the PMv as described in Materials and Methods. Therefore, it cannot be guaranteed that BDA was completely injected into the entire PMv region concerned with hand/arm movements. In addition, BDA can potentially spread into the regions for other body parts, including those for orofacial movements (Godschalk et al., 1995). In this study, we anatomically defined $\mathrm{PMv}$-h as the ventral bank of both the arcuate spur and the genu of the arcuate sulcus, as shown in a previous report (Dum and Strick, 1991), and calculated the unbiased volumes. The average percentages of the BDA injection core volume were $45.7 \pm 4.2 \%$ (mean \pm SE) and $32.4 \pm$ $3.2 \%$ of the total volume of $\mathrm{PMv}-\mathrm{h}$ in the intact and lesioned monkeys, respectively. A small amount of BDA injection core was also found outside the defined PMv-h but within the PMv $(15.5 \pm 7.5$ and $6.9 \pm 6.7 \%$ in intact and lesioned monkeys, respectively). Approximately $70 \%$ of the entire BDA injection core was distributed within the PMv-h. Therefore, BDA was mainly injected into the anatomically defined PMv-h, which has been considered to be involved in hand movements from both anatomical and electrophysiological observations (Dum and Strick, 1991; Godschalk et al., 1995; Stark et al., 2007; Murata et al., 2015b; Dea et al., 2016; Higo et al., 2016; Quessy et al., 2016). These results strongly suggest involvement of increased frontocerebellar projections in hand motor recovery.

The present findings suggest two possible compensatory network mechanisms during motor recovery as summarized in Figure 8. One possible explanation is a mechanism in which there is increased sensorimotor coupling (Fig. 8, blue line). During motor recovery, animals were required to reshape grasping using their disabled hand after motor injury as detailed in our previous study (Murata et al., 2015b). Notably, the current study found that BDA-positive axons from the ip-PMv were more often observed in the Aldoc-negative and middle of the ip-FN. The Aldoc- negative zone of the FN is functionally associated with parts of the somatosensory system, whereas the positive zone is associated with the oculomotor/vestibular system (for review, see Sugihara, 2011). The FN is known to receive abundant sensory inputs from the spinocerebellar tract. Therefore, the reorganized projections from the PMv to FN may be integrated with somatosensory information, potentially leading to correlation between the mechanisms for motor recovery and motor learning. This hypothesis is supported by our previous observations with non-invasive neuroimaging. Murata et al. (2015b) revealed that in animals who have recovered from motor injury there was recovery of task performance accompanied by an increase in functional connectivity between the contralesional cerebellum and malfunctional motor cortex, as well as a significant increase in task-related cerebral blood flow in the ventral premotor cortex and cerebellum (Murata et al., 2015b). Classical experiments with the motor injury model also support the idea that premotor reorganization is associated with compensation for somatosensory impairments after motor cortical damage (Dancause et al., 2005).

A second possible mechanism is that increased frontocerebellar projections may enhance motor commands from the cerebral cortex to the lower motor control system (Fig. 8, yellow line), although it is unlikely very efficient, considering the lack of ICMS effects on hand movement in our previous study (Murata et al., 2015b). Our data suggest that motor recovery accompanies the increase in direct projections from the ip-PMv to the ipsilesional cerebellar deep nuclei, especially the FN. The FN has output neurons that have axonal projections descending along the so-called fastigiospinal tract and ending in the contralateral cervical spinal cord (Batton et al., 1977; Asanuma et al., 1983; Takahashi et al., 1987; for review, see Liang et al., 2011). Therefore, the motor command generated in the PMv for movements of the contralateral limb may be conveyed to the ipsilateral FN, which 
may be transmitted to contralateral spinal motor neurons via fastigiospinal projections to execute the final motor output. Because fastigiospinal projections are known to be engaged in the control of proximal extremities, this hypothesis is in line with previous literature suggesting that motor recovery of distal extremities could be achieved by replacing distal motor systems with those of proximal extremities. Damage to the direct corticospinal tract involves conversion of the descending motor system from distal to proximal extremities by recruitment of the indirect corticospinal pathways, such as the cortico-rubro-spinal (Lawrence and Kuypers, 1965, 1968; Takenobu et al., 2014; Ishida et al., 2016) and cortico-reticulo-spinal pathways (Fregosi et al., 2018). As a next step, it would be important to elucidate the relay of projections from the nuclei to the spinal cord change in these indirect corticospinal pathways to engage the proximal motor systems in the control of distal extremities.

\section{Conclusion}

The current study provides evidence that the motor cortical lesion and long-term motor recovery model is accompanied by axonal rewiring of projections from the premotor to cerebellar nuclei. When the anterograde neural tracer, BDA, was injected into the ip-PMv, higher numbers of BDA-labeled boutons and axons were found in the ipsilesional deep cerebellar nuclei (fastigial, interposed, and dentate) of monkeys following motor recovery than in the intact monkeys. The projections from the ip-PMv were particularly visible in ip-FN neurons and were confirmed to be functional by colocalization of the synaptic markers, synaptophysin and VGLUT1. BDA-labeled terminals were mainly located in the middle of the FN, which is negative for Aldoc, and in the potential somatosensory region of the ip-FN.

These results suggest that adult primate rewiring can occur in large-scale connections (Fig. 8), and that the fronto-cerebellar connection may underlie the mechanism of sensorimotor coupling and motor command enhancement for long-term motor recovery. However, providing direct proof of this idea may require novel methods to evaluate specific projections such as the double-viral vector technique, which allows selective blockage of target pathways in primates (Kinoshita et al., 2012).

\section{References}

Apps R, Hawkes R (2009) Cerebellar cortical organization: a one-map hypothesis. Nat Rev Neurosci 10:670-681.

Asanuma C, Thach WT, Jones EG (1983) Brainstem and spinal projections of the deep cerebellar nuclei in the monkey, with observations on the brainstem projections of the dorsal column nuclei. Brain Res 286:299322.

Batton RR 3rd, Jayaraman A, Ruggiero D, Carpenter MB (1977) Fastigial efferent projections in the monkey: an autoradiographic study. J Comp Neurol 174:281-305.

Borra E, Belmalih A, Gerbella M, Rozzi S, Luppino G (2010) Projections of the hand field of the macaque ventral premotor area F5 to the brainstem and spinal cord. J Comp Neurol 518:2570-2591.

Brochu G, Maler L, Hawkes R (1990) Zebrin II: a polypeptide antigen expressed selectively by Purkinje cells reveals compartments in rat and fish cerebellum. J Comp Neurol 291:538-552.

Cai Y, Xiong K, Zhang XM, Cai H, Luo XG, Feng JC, Clough RW, Struble RG, Patrylo PR, Chu Y, Kordower JH, Yan XX (2010) $\beta$-Secretase-1 elevation in aged monkey and Alzheimer's disease human cerebral cortex occurs around the vasculature in partnership with multisystem axon terminal pathogenesis and beta-amyloid accumulation. Eur J Neurosci 32:1223-1238.

Chung SH, Marzban H, Hawkes R (2009) Compartmentation of the cerebellar nuclei of the mouse. Neuroscience 161:123-138.

Coffman KA, Dum RP, Strick PL (2011) Cerebellar vermis is a target of projections from the motor areas in the cerebral cortex. Proc Natl Acad Sci U S A 108:16068-16073.
Consalez GG, Hawkes R (2013) The compartmental restriction of cerebellar interneurons. Front Neural Circuits 6:123.

Dancause N, Barbay S, Frost SB, Plautz EJ, Chen D, Zoubina EV, Stowe AM, Nudo RJ (2005) Extensive cortical rewiring after brain injury. J Neurosci 25:10167-10179.

Dea M, Hamadjida A, Elgbeili G, Quessy S, Dancause N (2016) Different patterns of cortical inputs to subregions of the primary motor cortex hand representation in Cebus apella. Cereb Cortex 26:1747-1761.

De Zeeuw CI, Ten Brinke MM (2015) Motor learning and the cerebellum. Cold Spring Harb Perspect Biol 7:a021683.

Dum RP, Strick PL (1991) The origin of corticospinal projections from the premotor areas in the frontal lobe. J Neurosci 11:667-689.

Fregosi M, Contestabile A, Hamadjida A, Rouiller EM (2017) Corticobulbar projections from distinct motor cortical areas to the reticular formation in macaque monkeys. Eur J Neurosci 45:1379-1395.

Fregosi M, Contestabile A, Badoud S, Borgognon S, Cottet J, Brunet JF, Bloch J, Schwab ME, Rouiller EM (2018) Changes of motor corticobulbar projections following different lesion types affecting the central nervous system in adult macaque monkeys. Eur J Neurosci 48:2050-2070.

Friel KM, Nudo RJ (1998) Recovery of motor function after focal cortical injury in primates: compensatory movement patterns used during rehabilitative training. Somatosens Mot Res 15:173-189.

Frost SB, Barbay S, Friel KM, Plautz EJ, Nudo RJ (2003) Reorganization of remote cortical regions after ischemic brain injury: a potential substrate for stroke recovery. J Neurophysiol 89:3205-3214.

Glees P, Cole J (1950) Recovery of skilled motor functions after small repeated lesions of motor cortex in macaque. J Neurophysiol 13:137-148.

Godschalk M, Mitz AR, van Duin B, van der Burg H (1995) Somatotopy of monkey premotor cortex examined with microstimulation. Neurosci Res 23:269-279.

Higo N, Kunori N, Murata Y (2016) Neural activity during voluntary movements in each body representation of the intracortical microstimulationderived map in the macaque motor cortex. PLoS One 11:e0160720.

Ishida A, Isa K, Umeda T, Kobayashi K, Kobayashi K, Hida H, Isa T (2016) Causal link between the cortico-rubral pathway and functional recovery through forced impaired limb use in rats with stroke. J Neurosci 36:455467.

Ito M (2008) Control of mental activities by internal models in the cerebellum. Nat Rev Neurosci 9:304-313.

Johansen-Berg H, Dawes H, Guy C, Smith SM, Wade DT, Matthews PM (2002) Correlation between motor improvements and altered fMRI activity after rehabilitative therapy. Brain 125:2731-2742.

Kandel ER, Schwartz JH, Jessell TM (2012) Principles of neural science, Ed 5. New York: McGraw-Hill.

Kinoshita M, Matsui R, Kato S, Hasegawa T, Kasahara H, Isa K, Watakabe A, Yamamori T, Nishimura Y, Alstermark B, Watanabe D, Kobayashi K, Isa $\mathrm{T}$ (2012) Genetic dissection of the circuit for hand dexterity in primates. Nature 487:235-238.

Lawrence DG, Kuypers HG (1965) Pyramidal and non-pyramidal pathways in monkeys: anatomical and functional correlation. Science 148:973-975.

Lawrence DG, Kuypers HG (1968) The functional organization of the motor system in the monkey: I. The effects of bilateral pyramidal lesions. Brain 91:1-14.

Leyton AS, Sherrington CS (1917) Observations on the excitable cortex of the chimpanzee, orangutan, and gorilla. Exp Physiol 11:135-222.

Liang H, Paxinos G, Watson C (2011) Projections from the brain to the spinal cord in the mouse. Brain Struct Funct 215:159-186.

Liu Y, Rouiller EM (1999) Mechanisms of recovery of dexterity following unilateral lesion of the sensorimotor cortex in adult monkeys. Exp Brain Res 128:149-159.

Longley M, Yeo CH (2014) Distribution of neural plasticity in cerebellumdependent motor learning. Prog Brain Res 210:79-101.

Madigan JC, Carpenter MB (1971) Cerebellum of the rhesus monkey: atlas of lobules, laminae, and folia, in sections. Baltimore: University Park.

Mayhew TM (1992) A review of recent advances in stereology for quantifying neural structure. J Neurocytol 21:313-328.

Murata Y, Higo N, Oishi T, Yamashita A, Matsuda K, Hayashi M, Yamane S (2008) Effects of motor training on the recovery of manual dexterity after primary motor cortex lesion in macaque monkeys. J Neurophysiol 99: 773-786.

Murata Y, Higo N, Oishi T, Isa T (2015a) Increased expression of the 
growth-associated protein- 43 gene after primary motor cortex lesion in macaque monkeys. Neurosci Res 98:64-69.

Murata Y, Higo N, Hayashi T, Nishimura Y, Sugiyama Y, Oishi T, Tsukada H, Isa $\mathrm{T}$, Onoe $\mathrm{H}$ (2015b) Temporal plasticity involved in recovery from manual dexterity deficit after motor cortex lesion in macaque monkeys. J Neurosci 35:84-95.

Nudo RJ (2013) Recovery after brain injury: mechanisms and principles. Front Hum Neurosci 7:887.

Nudo RJ, Wise BM, SiFuentes F, Milliken GW (1996) Neural substrates for the effects of rehabilitative training on motor recovery after ischemic infarct. Science 272:1791-1794.

Passingham RE, Perry VH, Wilkinson F (1983) The long-term effects of removal of sensorimotor cortex in infant and adult rhesus monkeys. Brain 106:675-705.

Quessy S, Côté SL, Hamadjida A, Deffeyes J, Dancause N (2016) Modulatory effects of the ipsi and contralateral ventral premotor cortex (PMv) on the primary motor cortex (M1) outputs to intrinsic hand and forearm muscles in Cebus apella. Cereb Cortex 26:3905-3920.

Rizzolatti G, Luppino G (2001) The cortical motor system. Neuron 31: 889-901.

Rizzolatti G, Camarda R, Fogassi L, Gentilucci M, Luppino G, Matelli M (1988) Functional organization of inferior area 6 in the macaque monkey: II. Area F5 and the control of distal movements. Exp Brain Res 71: 491-507.

Saleem KS, Logothetis NK (2007) A combined MRI and histology atlas of the rhesus monkey brain in stereotaxic coordinates. London: Academic.

Saleem KS, Logothetis NK (2012) A combined MRI and histology atlas of the rhesus monkey brain in stereotaxic coordinates. London: Academic.

Sarna JR, Marzban H, Watanabe M, Hawkes R (2006) Complementary stripes of phospholipase $\mathrm{C} \beta 3$ and $\mathrm{C} \beta 4$ expression by Purkinje cell subsets in the mouse cerebellum. J Comp Neurol 496:303-313.

Seitz RJ, Höflich P, Binkofski F, Tellmann L, Herzog H, Freund HJ (1998) Role of the premotor cortex in recovery from middle cerebral artery infarction. Arch Neurol 55:1081-1088.

Small SL, Hlustik P, Noll DC, Genovese C, Solodkin A (2002) Cerebellar hemispheric activation ipsilateral to the paretic hand correlates with functional recovery after stroke. Brain 125:1544-1557.

Stanfield BB, O'Leary DD (1985a) Fetal occipital cortical neurons transplanted to the rostral cortex can extend and maintain a pyramidal tract axon. Nature 313:135-137.

Stanfield BB, O'Leary DD (1985b) The transient corticospinal projection from the occipital cortex during the postnatal development of the rat. J Comp Neurol 238:236-248.

Stanfield BB, O'Leary DD, Fricks C (1982) Selective collateral elimination in early postnatal development restricts cortical distribution of rat pyramidal tract neurones. Nature 298:371-373.

Stark E, Asher I, Abeles M (2007) Encoding of reach and grasp by single neurons in premotor cortex is independent of recording site. J Neurophysiol 97:3351-3364.

Sugihara I (2011) Compartmentalization of the deep cerebellar nuclei based on afferent projections and aldolase C expression. Cerebellum 10:449463.

Sugihara I, Shinoda Y (2004) Molecular, topographic, and functional organization of the cerebellar cortex: a study with combined aldolase $\mathrm{C}$ and olivocerebellar labeling. J Neurosci 24:8771-8785.

Sugihara I, Shinoda Y (2007) Molecular, topographic, and functional organization of the cerebellar nuclei: analysis by three-dimensional mapping of the olivonuclear projection and aldolase C labeling. J Neurosci 27:9696-9710.

Sugiyama Y, Higo N, Yoshino-Saito K, Murata Y, Nishimura Y, Oishi T, Isa T (2013) Effects of early versus late rehabilitative training on manual dexterity after corticospinal tract lesion in macaque monkeys. J Neurophysiol 109:2853-2865

Takahashi O, Satoda T, Matsushima R, Uemura-Sumi M, Mizuno N (1987) Distribution of cerebellar neurons projecting directly to the spinal cord: an HRP study in the Japanese monkey and the cat. J Hirnforsch 28:105-113.

Takenobu Y, Hayashi T, Moriwaki H, Nagatsuka K, Naritomi H, Fukuyama $\mathrm{H}$ (2014) Motor recovery and microstructural change in rubro-spinal tract in subcortical stroke. Neuroimage Clin 4:201-208.

Taub E, Uswatte G, Elbert T (2002) New treatments in neurorehabilitation founded on basic research. Nat Rev Neurosci 3:228-236.

Travis AM (1955) Neurological deficiencies after ablation of the precentral motor area in Macaca mulatta. Brain 78:155-173.

Vilensky JA, Gilman S (2002) Lesions of the precentral gyrus in nonhuman primates: a pre-Medline bibliography. Int J Primatol 23:1319-1333.

Yamamoto T, Oishi T, Higo N, Murayama S, Sato A, Takashima I, Sugiyama Y, Nishimura Y, Murata Y, Yoshino-Saito K, Isa T, Kojima T (2013) Differential expression of secreted phosphoprotein 1 in the motor cortex among primate species and during postnatal development and functional recovery. PLoS One 8:e65701. 